\title{
HUMAN CHROMOSOME MAP OF LYMPHEDEMA-LYMIPHANGIOGENESIS GENES: TEMPLATE FOR CURRENT AND FUTURE DISCOVERY
}

\author{
M.H. Witte, R.P. Erickson, L. Luy, P. Brouillard, M. Vikkula
}

Departments of Surgery, Neurosurgery, and Pediatrics (MHW,RPE,LL), University of Arizona, Tucson, Arizona, USA; Human Molecular Genetics (PB,MV), de Duve Institute, University of Louvain, Brussels, Belgium; VASCERN VASCA European Reference Centre (MV), Center for Vascular Anomalies, Division of Plastic Surgery, University Clinics Saint-Luc, University of Louvain, Brussels; and Walloon Excellence in Lifesciences and Biotechnology (WELBIO), de Duve Institute (MV), University of Louvain, Brussels, Belgium

\section{ABSTRACT}

We have created a human chromosomal map of the location of known and candidate genes involved in primary lymphedema (PLE). This should facilitate further discovery and provide a basis for understanding microdeletions which cause lymphedema.

Keywords: primary lymphedema, chromosomes, genes, mutations

Beginning in 2000, as an outgrowth of the Human Genome Project, multiple pathogenic mutations and their transcribed proteins have been reported to underlie human familial lymphedema, lymphatic malformations, and pleiomorphic syndromic lymphatic disorders. These "lymphedema-lymphangiogenesis" factors have consisted of growth factor receptors and their ligands, transcription factors, endothelial junctional components, among others that have been implicated in lymphatic system structure and function. In addition, many more candidate genes have been pinpointed in mouse models of these human lymphatic disorders although the human counterparts have not yet been reported. To catalog and organize these disparate discoveries, we have created a human chromosome map from a comprehensive review of the existing literature to date to record, locate, and explore interactions of currently identified lymphatic disease-causing mutations and provide a template for future discovery of lymphedema-lymphangiogenesis genes and proteins.

\section{MATERIALS AND METHODS}

We have searched several databases, each with the different terms: 1) Online Mendelian Inheritance in Man (OMIM-97 entries for "lymphedema," finding many entries for case descriptions which have not been proven to be inherited, many entries for syndromes with only occasional lymphedema), and 2) Google Scholar with the terms "lymphedema and genes" (over 21,000 entries) and "lymphedema and mutations" (about 16,000 entries)-in both cases with many repeats, many multiple papers on the same gene, and mostly papers which are not about inherited conditions.

\section{RESULTS}

The known human or mouse genes which are associated with primary lymphedema, including complex lymphatic anomalies, 


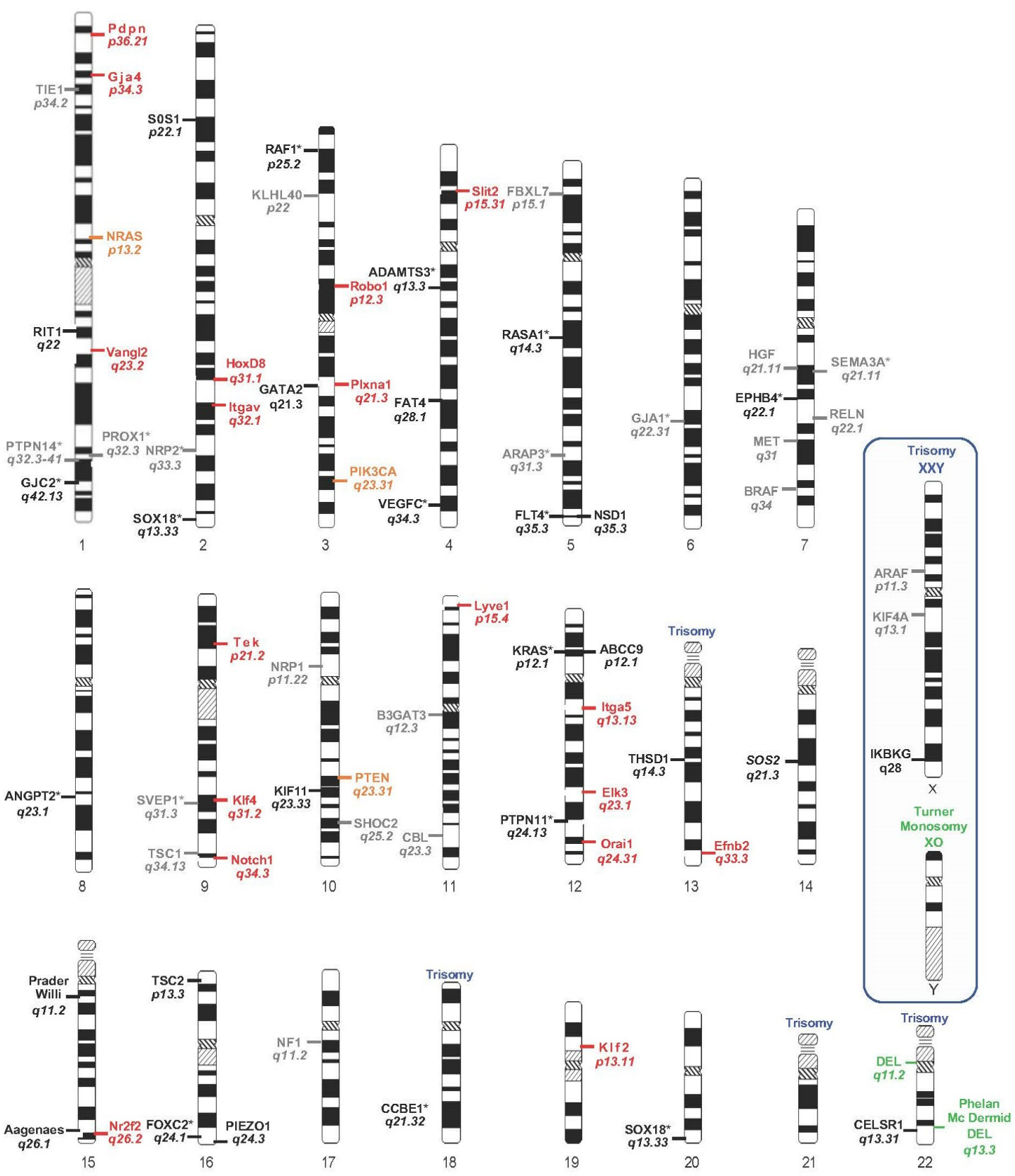

Human Chromosome Map depicting locations of gene variants and loci known to be associated with human primary lymphedema/lymphatic dysplasia syndromes, as well as candidate genes on the basis of murine studies. Black: location of confirmed human gene variants; grey: potential PLE genes awaiting further confirmation; orange: genes causing complicated lymphatic anomalies (CLAs); red: corresponding human location of mouse gene variants associated with lymphatic maldevelopment (i.e., "candidate genes" not yet identified in human primary lymphedema); *: pathogenic human gene variants with corresponding mouse models; green: location of genetic deletions; blue: chromosomal duplications associated with human PLE. See text and reference 1 for further details. 
are presented in Fig. It comprises 26 confirmed genes and five confirmed loci (including the two deletions on chromosome 22 and Turner syndrome's monosomy $\mathrm{X}$ ), as well as 18 potential PLE genes in which variants considered as potential mutations await confirmation by identification of additional cases and/or functional validation in vitro or in vivo. References for the locations can be found in Brouillard et al (1). Four recent additional potential PLE genes have been added.

\section{DISCUSSION}

Initially, because of closely linked genes in pathways in bacteria (Operons, e.g., Betagalactosidase), there were extensive generally unsuccessful searches for linkage of genes involved in pathways in higher organisms. Exceptions were the many closely linked genes in the major histocompatibility genes, $\mathrm{H}$-2 in mouse, $H L A$ in man. It is interesting to note that whereas if the 74 loci depicted were randomly dispersed, we would expect an average distance of about 49 megabases $(\mathrm{mb})$ between them. However, there are 3 cases of much closer linkage, although at distances compatible with hundreds of genes intervening: PROX1 and PTPN14 at $0.3 \mathrm{mb}$, FOXC2 and PIEZO1 at $2.2 \mathrm{mb}$, and FLT4 and NSD1 at $3.3 \mathrm{mb}$. It is interesting in this regard that a recent report described a large multigeneration family with highly penetrant primary lymphedema caused by various combinations of dominant $F O X C 2$ and recessive biallelic PIEZO1 mutations (2). There are 2 other linkages at about $5 \mathrm{mb}$ : GATA2 and PLXNA1 (3), and KIF11 and PTEN (4). These are likely to just be stochastic variations but might suggest further searches for chromatin configuration involvement in the expression of lymphedema genes. Due to close proximity of the two genes in these loci, and thus the low frequency of recombinations between them, the digenic variants could still follow dominant inheritance pattern.

\section{CONFLICT OF INTEREST AND DISCLOSURE STATEMENT}

All authors declare no financial compet- itive conflict of interest.

\section{ACKNOWLEDGMENTS}

Research support from University of Arizona Health Sciences Translational Imaging Program Projects Stimulus (TIPPS) Award (MHW); National Institutes of Health NHLBI R25HL108837 for diverse undergraduate research trainees (MHW, LL); MV's laboratories were financially supported by the Fonds de la Recherche Scientifique - FNRS Grants T.002 6.14 and T.0247.19, the Fund Generet managed by the King Baudouin Foundation (Grant 2018-J1810250-211305), and by la Région wallonne dans le cadre du financement de l'axe stratégique FRFS-WELBIO (WELBIOCR-2019C-06). MV has also received funding by the MSCA-ITN network V.A. Cure No 814316 and the Lymphatic Malformation Institute (LMI), USA.

\section{REFERENCES}

1. Brouillard, $\mathrm{P}, \mathrm{MH}$ Witte, RP Erickson, et al: Primary lymphoedema. Nat. Rev. Dis. Primers 7: 77, 2021. https://doi.org/ 10.1038/ s41572-021-00309-7

2. Mustacich, DJ, L Lei, MJ Bernas, et al: Digenic inheritance of a $F O X C 2$ mutation and two PIEZO1 mutations underlies congenital lymphedema in a multigeneration family. Am. J. Med. online prior to print. doi: https ://doi.org/ 10.1016/j.amjmed.2021.09.007

3. Bouvrée, K, I Brunet, R Del Toro, et al: Semaphorin3A, Neuropilin-1, and PlexinA1 are required for lymphatic valve formation. Circ. Res. 111:437-445, 2012. doi:10.1161/CIRCRESAHA. 112.269316.

4. Hopman, SM, RR Van Rijn, C Eng, et al: PTEN hamartoma tumor syndrome and Gorham-Stout phenomenon. Am. J. Med. Genet. A. 158A:1719-23, 2012. doi: 10.1002/ajmg.a.35406. Epub 2012 May 24. PMID: 22628360.

Marlys H. Witte, MD
Professor of Surgery, Neurosurgery, and
Pediatrics
University of Arizona College of Medicine
Tucson, AZ USA
Telephone: (520) 626-6118
E-mail: lymph@surgery.arizona.edu

Marlys H. Witte, MD

Professor of Surgery, Neurosurgery, and Pediatrics

University of Arizona College of Medicine

Tucson, AZ USA

E-mail: lymph@surgery.arizona.edu 